\title{
The Role of Orthotic Service in Modern Rehabilitation of Patients with Charcot-Marie-Tooth Disease
}

\section{Olga V. Petryaeva1, Natalia A. Shnayder ${ }^{1,2}$, Ivan P. Artyukhov ${ }^{1}$, Margarita R. Sapronova1, Irina O. Loginova ${ }^{1}$}

${ }^{1}$ V.F. Voyno-Yasenetsky Krasnoyarsk State Medical University, Krasnoyarsk, Russia

${ }^{2}$ V.M. Bechterev National Medical Research Center of Psychiatry and Neurology, Sankt-Petersburg, Russia

Email: olkalog@mail.ru

How to cite this paper: Petryaeva, O.V., Shnayder, N.A., Artyukhov, I.P., Sapronova, M.R. and Loginova, I.O. (2018) The Role of Orthotic Service in Modern Rehabilitation of Patients with Charcot-Marie-Tooth Disease. Journal of Biosciences and Medicines, 6, 23-34.

https://doi.org/10.4236/jbm.2018.67003

Received: June 19, 2018

Accepted: July 21, 2018

Published: July 24, 2018

Copyright (c) 2018 by authors and Scientific Research Publishing Inc. This work is licensed under the Creative Commons Attribution International License (CC BY 4.0).

http://creativecommons.org/licenses/by/4.0/

(c) (i) Open Access

\begin{abstract}
Charcot-Marie-Tooth (CMT) disease, which encompasses several hereditary motor and sensory neuropathies, is one of the most common neuromuscular disorders. $80 \%$ of patients having CMT disease are diagnosed with per cavus deformity. Orthosis is widespread and varies widely in forms. The paper arises the necessity of habilitation at the earliest possible stage as only a few patients use it. The meta-analysis of 412 scientific papers concerning this problem demonstrates the getting better gate, balance and the stopping CMT progression which is scientifically proven. It is also shown that patients with CMT use low prevalence of orthotics, and demonstrate low compliance of patients (for various reasons), high expectations from this habilitation technique.
\end{abstract}

\section{Keywords}

Charcot-Marie-Tooth Disease (CMT), Habilitation, Rehabilitation, Hereditary Sensori-Motor Neuropathies (HSMN), Contractures, Orthosis, Demyelinating Diseases (DMD), Orthotic Management, Ankle-Foot Orthoses (AFOs)

\section{Introduction}

Charcot-Marie-Tooth (CMT) disease, which encompasses several hereditary motor and sensory neuropathies, is one of the most common neuromuscular disorders. The molecular genotypes of CMT and the resultant clinical and electrophysiological phenotypes have increased greatly in the past decade. Characterized by electrodiagnostic studies into demyelinating (type 1) and axonal (type 2) forms, subsequent genetic testing often provides an exact diagnosis of a spe- 
cific subtype of CMT [1].

According to Johnson NE et al., the most prevalent themes were: foot and ankle weakness (99.7\%), impaired balance (98.6\%), limitations with mobility (97.5\%), hand and finger weakness (97.0\%) [2]. CMT is characterized by distal sensory loss, skeletal deformities (e.g., pes cavus), and also decrease or absence of tendon reflexes. The most frequent findings are ataxia, hypoesthesia and loss of vibration [3]. Disease onset usually occurs during the first or second decade of life and can be described as slowly progressive, but the patient's life span seems normal. CMT is also the most common neuromuscular cause of foot deformity among children, and occult CMT is frequently the cause of abnormal feet among adults. Hand deformities tend to occur in adults but can also be found in severe childhood neuropathy. The foot abnormality is usually a cavovarus deformity, which is a complex deformity of the forefoot and hindfoot. The hindfoot varus deformity is usually secondary to the forefoot pronation, and early orthotic treatment and physiotherapy may delay the onset of fixed hindfoot deformities. Many other foot deformities can arise as many patients are tested lately [4]. The study of So Young Joo et al. confirms that the lateral talo-first metatarsal angle, anteroposterior talo-first metatarsal angle, lateral calcaneal-first metatarsal angle, lateral naviocuboid overlap, lateral calcaneal pitch, lateral tibiocalcaneal angle, and lateral talocalcaneal angle in the group of CMT patients showed a significant difference compared to the normal control group of healthy people [5]. It confirms that the diagnosis CMT is most associated with pes cavus [6].

Foot morphology in the youngest children with initially CMT is pes planovalgus, with the minority being pes cavovarus. As children are growing, the proportion changes are becoming nearly entirely cavus or cavovarus, with very few remaining planovalgus or planus [7]. Two thirds of adults with symptomatic cavus foot have an underlying neurological condition [8]. The frequency of patients' amount who were bilateral cavovarus feet being diagnosed with CMT is about $78 \%$ [9].

There are no pharmacologic cures for CMT. A well-balanced diet and weight control can help minimize disability. Among patients with CMT disease, according to Wicart P, $80 \%$ have pes cavus deformity [10]. According to Laura $\mathrm{M}$ et al., foot deformities were diagnosed in $71 \%$ of CMT patients; $30 \%$ of the patients had surgery invention [11].

Using orthosis during a conservative treatment can help preservation of patients with CMT life quality.

Modern orthosis is a large branch, based on the latest achievements in the development of materials and modules to satisfy the desired therapeutic or prophylactic effect. The tasks of orthosis include not only the limbs fixation, for example, with a fracture, but also the ability to flex the joints, restore the lost function of the musculoskeletal system, or replace it.

Orthoses aimed at offering structural support to focal, and/or global ankle and foot weakness may range from simple shoe inserts to complete AFO bracing, 
may be plastic or elastic, or may be fabricated from durable plastics to custom carbon-fiber composites [12].

Ankle-foot orthotics (AFOs) or nighttime resting splints have been used to maintain a $90^{\circ}$ angle of the foot relative to the tibia. Some authors indicate that such splinting is effective for reducing heel cord contractures in demyelinating diseases (DMD). However, other investigators do not believe that AFOs change the natural history of heel cord contracture formation. Foot deformities are common in peripheral neuropathies such as CMT. Treatment of foot deformities depends on the patient's age, foot flexibility, bony deformity, and muscle imbalance. A nighttime or full-time AFO in a neutral ankle position custom molded to the foot deformity may decrease the tendency toward further development of the deformity. A supple foot can be managed non-operatively by a solid ankle AFO in the neutral position [13].

Orthopaedic treatment is aimed at preventing the progression of deformities and providing stability to the skeletal system to improve children and adult quality of the life [14].

The objective is to attempt the meta-analysis which is performed to assess the role of orthotic management in habilitation of patients with Charcot-Marie-Tooth disease.

\section{Methods}

The design of the presented paper demanded the huge analysis of scientific resources which involved such sources as PubMed, MedLine, Web of Science Core Collection, Web of Science, Russian Science Citation Index, KCI-Korean Journal Database. The most significant are presented in Table 1.

The search was limited to the period since 2008 till 2018 (10 years). We analyzed available full-text articles and abstracts published in English and Russian. The search was conducted in 2 stages: 1-searching for articles on the keyword "orthosis". The total amount of the found manuscripts is 4561: Web of Science (2227), MEDLINE (2018), PUBMED (278), KCI-Korean Journal Database (34), Russian Science Citation Index (4) (Figure 1). Of these, the distribution by year 2008 (140), 2009 (172), 2010 (182), 2011 (201), 2012 (220), 2013 (315), 2014 (353), 2015 (324), 2016 (360), 2017 (335) (Figure 2).

Table 1. Databases used in this meta-analysis.

\begin{tabular}{ccc}
\hline \multirow{2}{*}{ Data base } & \multicolumn{2}{c}{ Articles that meet the inclusion/exclusion criteria } \\
\cline { 2 - 3 } & Found & Included \\
\hline PubMed & 129 & 13 \\
MedLine & 10 & 6 \\
Web of Science Core Collection & 235 & 13 \\
Web of Science Russian Citation Index & 4 & 1 \\
KCI-Korean Journal Database & 34 & \\
\hline
\end{tabular}




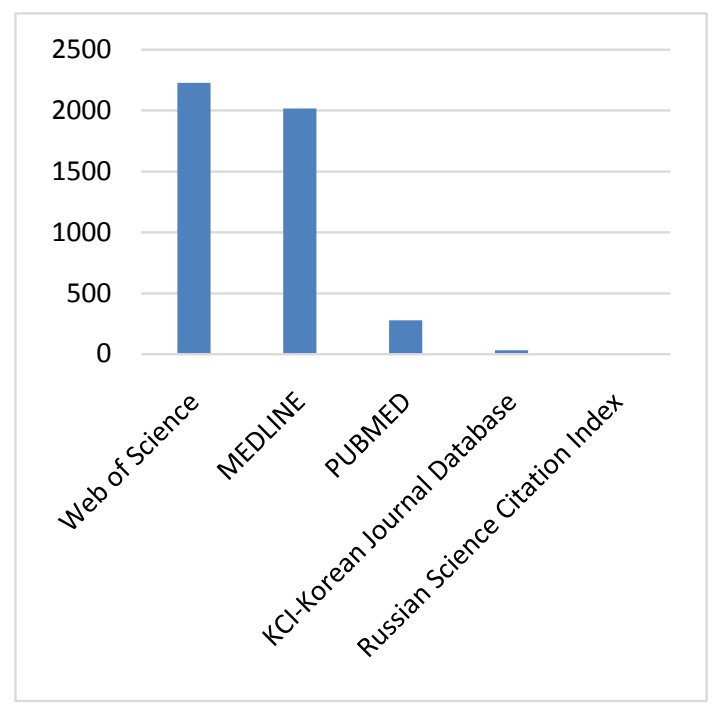

Figure 1. Number of articles in the analyzed databases.

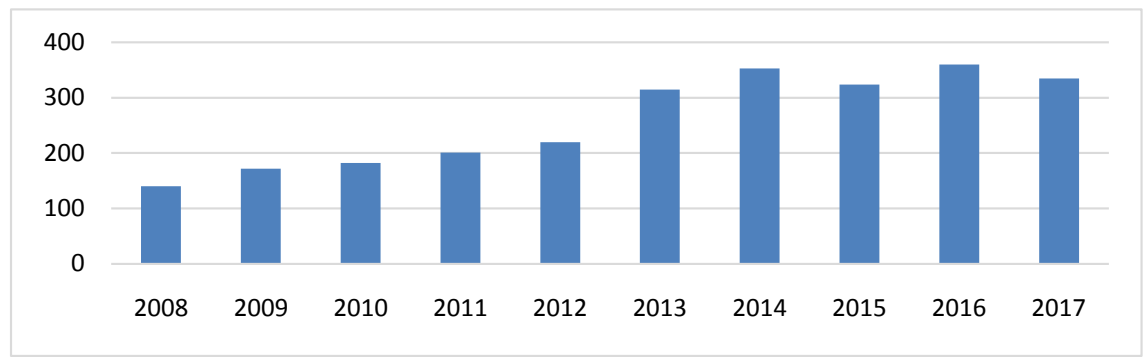

Figure 2. The distribution of the articles studied by the year of publication.

Stage 2-filtering search results using the keywords "Charcot-Marie-Tooth disease", "CMT", "hereditary sensori-motor neuropathies", "HSMN". The distribution of scientific papers remained the same for years. The number of articles had decreased significantly: 2008 (2), 2009 (1), 2010 (2), 2011 (3), 2012 (5), 2013 (-), 2014 (4), 2015 (3), 2016 (4), 2017 (2) (Figure 3). Most works were in English.

\section{Results}

On the basis of the analyzed scientific works, it is possible to distinguish various types of orthoses (Table 2).

1) Effectiveness of orthotics in CMT.

The study of Guillebastre B et al. describes the comparison of orthopedic shoes, plastic AFOs and elastic AFOs. The result was evaluated by Gait analysis and Yoden Index. The results showed that AFO prescription is relevant for improving walking balance and performance [15].

The study describing comparison of the effects on teenagers with CMT, some of whom used night orthoses for 4 weeks, and the other part conducted stretching sessions in equal period. The reflected result is as follows: the night orthosis improved the dorsiflexion of the ankle by 4 degrees; after 4 weeks of 
Table 2. Orthoses on the lower extremities used in medical practice.
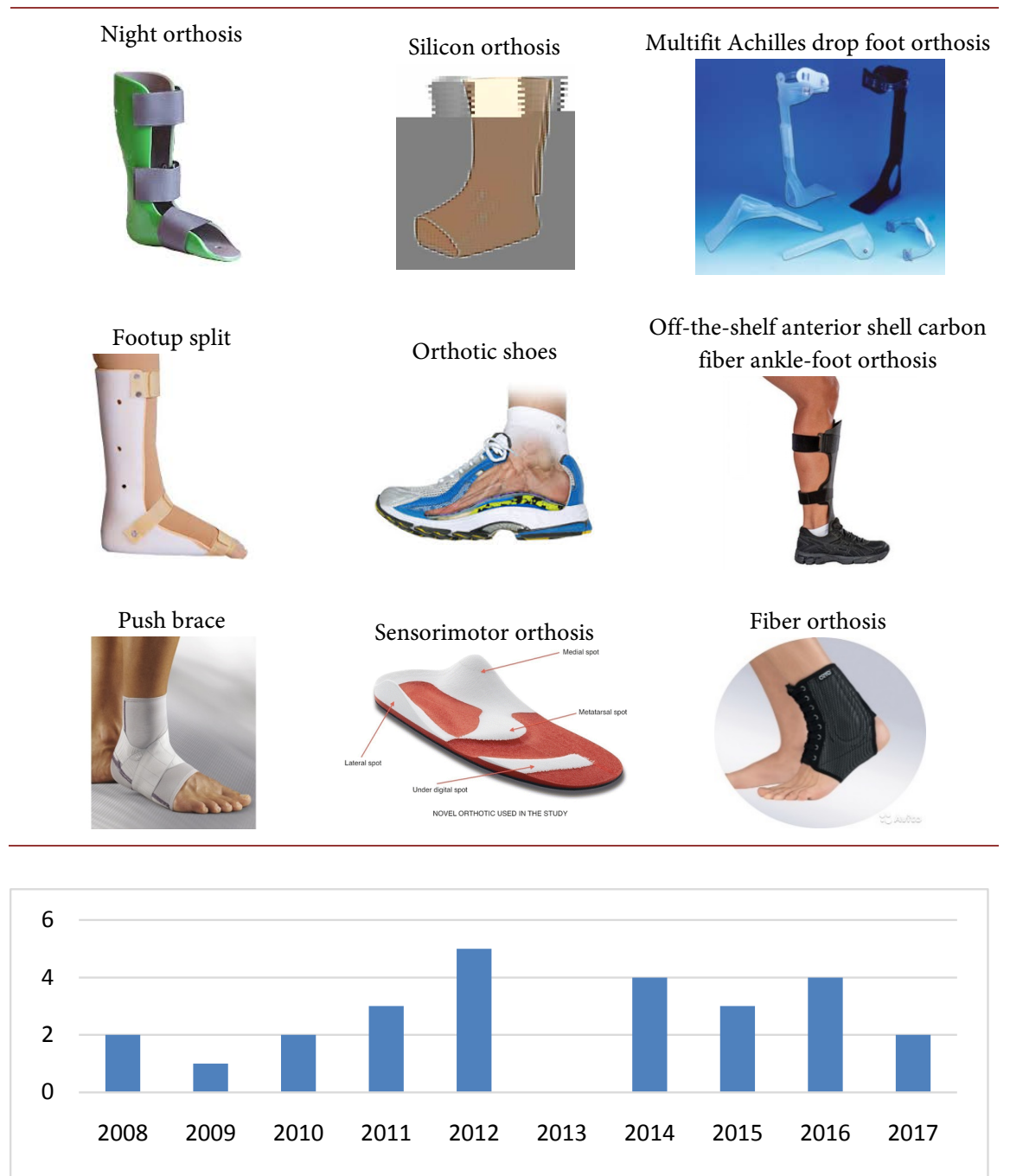

Figure 3. The distribution of articles included in the meta-analysis by years of publication.

stretching, the dorsiflexion improved by 3 degrees that original range (Rose KJ et al.) [16].

Ramdharry GM et al. presented various types of orthoses ("Footup splint", "Push brace" and Multifit Achilles drop foot orthosis) and compared their effectiveness. The authors showed that no significant differences were registered in the speed and step length of patients wearing AFOs and patients wearing standard shoes. The authors pointed out a significant influence of the AFOs on hip flexion at the oscillating stage of the step, and on the dorsiflexion of the ankle; this prevented the foot from falling and reduced the tripping frequency [17].

Kozhevnikov OV et al. conducted a comparative study of the use of conservative treatment combining exercise therapy, physiotherapy and orthotics, and complex, operational. Results were evaluated after 6 months and 4 years. The conclusion of this study demonstrates that conservative treatment gives stabili- 
zation of the condition at both control time points, and the complex treatment promotes restoration of bone-joint relations, ensured functional consistency [18].

Mnatsakanian A et al. conducted a study on convenience and effectiveness new off-the-shelf anterior shell carbon fiber ankle-foot orthoses (CFAFOs) on 123 patients. By the obtained dates, 109 of 123 (89\%) were satisfied with this orthoses. Anterior shell CFAFOs should be considered for most neuromuscular patients with distal leg weakness [19].

However, not all studies have such excellent results-Uygur F did not have any result of using 90 degrees of orthoses for the whole night. This makes one think about the correct and necessary use of orthosis in people with CMT [20]. According Anens E et al. qualitative content analysis revealed that personal factors such as fatigue, poor balance, muscle weakness, and pain were important barriers for physical activity behavior. Facilitators of physical activity were self-efficacy for physical activity, activity-related factors, and assistive devices [21].

2) Influence of orthosis on balance and gait.

The most urgent problems among CMT patients are: factors associated with functional ankle instability-female gender, ipsilateral cavus foot posture, toe-to-heel balance and contralateral ankle instability [22]. The study of Phillipset MF et al. was conducted on the effect of various types of orthoses on the gait rate of patients with CMT. The result was gait velocity. It was greatest wearing polypropylene orthoses, median 0.96 (interquartile range (IQR) 0.75 - 1.18) $\mathrm{ms}$ $(-1)$, compared with silicone orthoses, median $0.88(0.71-1.12) \mathrm{ms}(-1)$, and no orthosis, median $0.79(0.56-0.84) \mathrm{ms}(-1), P=0.006$. The silicone orthoses met goals more successfully and scored more favourably for comfort, 5.0 (5.0 - 6.0), $P=0.003$ and pain, $5.5(4.0-7.0), P=0.015$. Future modifications to study methodology were identified, such as a longer period of wear and measurement of walking in different situations [23].

Guillebastre Bet et al. demonstrated us that compared with ordinary shoes alone, adding plastic ankle-foot orthoses partially improved both gait and posture control. In the study it was included 26 patients with CMT. The author presented ankle-foot orthosis prescription appears relevant for improving balance and gait performance in patients with CMT, particularly when the model adequately compensates for specific muscle deficits [15].

In our study, we found that AFOs can help reduce foot drop and reduce proximal compensatory movements while walking by people with CMT. This suggests, however, that some AFOs may reduce the function of the ankle plantarflexors depending on the material properties [17].

In study of Wegener C et al. a new type of orthosis was submitted. It is a sensorimotor orthosis which increased medial ground reaction forces and increase pressure at the heel, midfoot and toes. Sensorimotor orthoses produce changes in kinematic, kinetic and pressure variables in adults with CMT and are re- 
garded as more comfortable, cushioned and stable during walking [24]. An ideal orthotic for a subtle cavus foot should support the lateral hindfoot and midfoot with a wedge [25].

Orthoses can be used not only for patients with CMT. Orthoses are universal things for rehabilitation patients with all neuromuscular diseases. The study of Ortlieb A et al. investigates the design of assistive orthoses specifications with the goal of adding month or year of near normal mobility for patients with muscle weakness (facio scapula humeral muscular dystrophy, CMT, Becker muscular dystrophy, myotonic dystrophy, inclusion body myositis). The results highlight the effect of muscle weakness on gait pattern and walking velocity [26].

While searching the scientific works on patients with CMT management and orthopedic manifestations, a large number of works on operative stop treatment [6] [27] [28] [29], where high results of these operations were proved, were revealed. However, there should always be cautious in prescribing such a radical treatment [30], also for neuromuscular diseases, in the cases when surgery is not the final treatment, it is also necessary to conduct long-term postoperative facilitation and, in one way or another, training in a new way of life and sports regimen. This brings us back to conservative treatment again.

3) Patients' compliance to orthotics

According to Chetlin RD et al. 87\% patients do exercises at home, if they do not need additional equipment [31]. But as soon as there is a need to change the established routine of life, the compliance of patients is sharply reduced. For example, in a study by Vinci et al., 25 patients received recommendations on the use of orthoses and only 5 of them started to be used. And 3/5 patients "hated" them [32].

\section{Discussion}

Problems of Orthotic Management:

1) Division of definitions: Rehabilitation or Habilitation?

Habilitation is the preparation for a certain type of activity, the standard of medical care and psychosocial interventions for the adaptation of patients (social, domestic, professional). In general, habilitation is a comprehensive approach (behavioral, non-pharmacological, medicamentous) to caring for people with progressive hereditary diseases and congenital malformations. Rehabilitation also returns patients to an earlier, higher level of functioning (for example, in the case of stroke or traumatic neuropathy) [33]. In this article, we prefer to use the word "habilitation" and encourage colleagues to do so.

2) The need for a dynamic replacement of approaches to the selection of orthoses in the progression of the disease.

As it can be seen from Table 2, at present time there is a wide variety of orthoses. Depending on the neurological pattern and the presence of walking function violations at the time of orthoses selection, one of the suggested is chosen. It is also necessary to take into account the degree and rate of progression of CMT, 
this disease is heterogeneous and the different types of CMT occur in different ways. Thus, for example, the case of CMT $2 S$ type in a young man of 20 years old with the initial signs of the disease [34] and the case of CMT 4C type in a girl of 10 years with a gradient course and highly disabling symptoms were described [35].

3) Availability of orthoses in different countries.

The availability of orthoses in different countries varies but remains at a sufficient level so patients can receive it. But there is another no less important problem-many patients do not know that they can use. Currently, the situation is changing for the better, for example, the information specialized Internet resources are developing, as well as the individual sites or groups in social networks where patients can communicate and share information among themselves, which raises awareness of orthesis among the consumer [36]-[41].

4) Low compliance of patients with CMT to orthotics.

Low compliance is another acute problem. Many authors wrote on this topic, for example, Danilov believes that this problem arose because of the lack of therapeutic interaction between the doctor and the patient [42]. Several authors came to the conclusion that the patient's compliance is formed at a doctor's appointment, it is very important to form a correct perception of therapeutic interventions and adequate expectations of the patient [43] [44]. Also, the "white coat phenomenon" determines the desired interval between appointments by the doctor [45].

\section{Conclusions}

It is well known that one of the gold rules for managing patients with CMT is the beginning of habilitation at the earliest possible stage as many of the symptoms have the greatest increase in prevalence and impact in the first decade of symptoms. Future therapeutic trials may consider early intervention as optimal given the apparent rapidity of progression in the first decade after first symptom occurrence. Patients with any DMD are susceptible to the development of contractures and scoliosis. Stretching exercises and nightly bracing can help to prevent the contractures from becoming severe. At the age of $8-15$, children with DMD have a sensation of the knees locking. It is easier to keep children with DMD walking than to induce walking once they have stopped. Therefore, contractures should be treated early to maintain ambulation, because, if it is maintained even for 1 - 2 years, it considerably benefits these children and delays the inevitable development of scoliosis and kyphosis. Proceeding from the foregoing, we come to the conclusion that orthosis is an indispensable method of habilitation of patients with any HSMN. We and other our colleagues prefer to think that it is necessary to use a multidisciplinary approach to create a universal and effective patient management model, which includes a neurologist, orthopedist, physiotherapist, therapist for the treatment of comorbidities. And also, according to Schnayder NA et al., clinical psychologists help patients in the per- 
ception of the internal picture of the disease and make changes in their own body [33] [46] [47] [48].

We call to respect alternating treatment in a polyclinic and specialized rehabilitation center because here it is possible not only to eliminate deformities, improve joint mobility, correctly and timely supply the patient with prosthetic and orthopedic products, but also to develop the most appropriate ways to compensate for impaired functions. Communication with the attending physician should lead to an increase in the patient's motivation for treatment and the development of an active own life position. Periodic monitoring by the doctor and availability of communication with medical workers at any time are also the basis of the patient's compliance in independent work on the body and maintaining the necessary level of physical activity.

Improving the pattern of walking, gait speed, reducing pain, prolonged preservation of limbs without serious orthopedic pathologies, and therefore a longer possibility to follow their usual lifestyle, all can improve the patient's quality of life and keep it at a high level.

\section{References}

[1] El-Abassi, R., England, J.D. and Carter, G.T. (2014) Charcot-Marie-Tooth Disease: An Overview of Genotypes, Phenotypes, and Clinical Management Strategies. PM \& $R, 4,342-355$. https://doi.org/10.1016/j.pmrj.2013.08.611

[2] Johnson, N.E., Heatwole, C.R., Dilek, N., Sowden, J., Kirk, C.A., Shereff, D., Shy, M.E. and Herrmann, D.N. (2014) On Behalf of the Inherited Neuropathies Consortium. Quality-of-Life in Charcot Marie Tooth Disease: The Patient's Perspective. Neuromuscular Disorder, 11, 1018-1023. https://doi.org/10.1016/j.nmd.2014.06.433

[3] Piazza, S., Ricci, G., CaldarazzoIenco, E., Carlesi, C., Volpi, L., Siciliano, G. and Mancuso, M. (2010) Pes Cavus and Hereditary Neuropathies: When a Relationship Should Be Suspected. Journal of Orthopaedics and Traumatology, 11, 195-201. https://doi.org/10.1007/s10195-010-0114-y

[4] Houlden, H., Charlton, P. and Singh, D. (2007) Neurology and Orthopaedic. Journal of Neuralology, Neurosurgery, and Psychiatry, 78, 224-232. https://doi.org/10.1136/jnnp.2006.092072

[5] Joo, S.Y., Choi, B.-O., Kim, D.Y., Jung, S.J., Cho, S.Y. and Hwang, S.J. (2011) Foot Deformity in Charcot Marie Tooth Disease according to Disease Severity. Annals of Rehabilitation Medicine, 4, 499-506.

[6] Maranho, D.A. and Volpon, J.B. (2009) Acquired Res Cavus in Charcoy-Marie-Tooth Disease. Revista Brasileira de Orthopedia, 6, 479-486.

https://doi.org/10.1590/S0102-36162009000600004

[7] Hoellwarth, J.S., Mahan, S.T. and Spencer, S.A. (2012) Painful Pes Planovalgus: An Uncommon Pediatric Orthopedic Presentation of Charcot-Marie-Tooth Disease. Journal of Pediatric Orthopaedic B, 5, 428-433. https://doi.org/10.1097/BPB.0b013e3283563750

[8] Alexander, I.J. and Johnson, K.A. (1989) Assessment and Management of Per Cavus in Charcout-Marie-Tooth Disease. Clinical Orthopaedics and Related Research, 246, 273-281.

[9] Nagai, M.K., Chan, G., Guille, J.T., Kumar, S.J., Scavina, M. and Mackenzie, W.G. 
(2006) Prevalence of Charcout-Marie-Tooth Disease in Patients Who Have Bilateral Cavovarus Feet. Journal of Pediatric Orthopaedics, 4, 438-443. https://doi.org/10.1097/01.bpo.0000226278.16449.c4

[10] Wicart, P. (2012) Cavus Foot, From Neonates to Adolescents. Orthopaedics and Traumatology: Surgery and Research, 98, 813-828. https://doi.org/10.1016/j.otsr.2012.09.003

[11] Laurá, M., Singh, D., Ramdharry, G., Morrow, J., Skorupinska, M., Pareyson, D., Burns, J., Lewis, R.A., Scherer, S.S., Herrmann, D.N., Cullen, N., Bradish, C., Gaiani, L., Martinelli, N., Gibbons, P., Pfeffer, G., Phisitkul, P., Wapner, K., Sanders, J., Flemister, S., Shy, M.E., Reilly, M.M., Inherited Neuropathies Consortium. (2018) Prevalence and Orthopedic Management of Foot and Ankle Deformities in Charcot-Marie-Tooth Disease. Muscle Nerve, 57, 255-259.

https://doi.org/10.1002/mus.25724

[12] McCorquodale, D., Pucillo, E.M. and Johnson, N.E. (2016) Management of Charcout-Marie-Tooth Disease: Improving Long-Term Care with a Multidisciplinary Approach. Journal of Multidisciplinary Healthcare, 9, 7-11.

[13] Skalsky, A.J. and McDonald, C.M. (2012) Prevention and Management of Limb Contractures in Neuromuscular Disease. Physical Medicine \& Rehabilitation Clinics of North America, 3, 675-687. https://doi.org/10.1016/j.pmr.2012.06.009

[14] Birch, J.G. (1998) Orthopedic Management of Neuromuscular Disorders in Children. Seminars in Pediatric Neurology, 5, 78-91. https://doi.org/10.1016/S1071-9091(98)80024-7

[15] Guillebaste, B., Calmels, P. and Rougier, P.R. (2011) Assessment of Appropriate Ankle-Foot Orthoses Models for Patient with Charcout-Marie-Tooth Disease. American Journal of Physical Medicine \& Rehabilitation, 90, 619-627. https://doi.org/10.1097/PHM.0b013e31821f7172

[16] Rose, K.J., Raymond, J., Refshauge, K., North, K.N. and Burns, J. (2010) Serial Nights Casting Increases Ankle Dorsiflexion Range in Children and Young Adults with Charcout-Marie-Tooth Disease: A Randomized Trial. Journal of Psysiotherapy, 56, 113-119. https://doi.org/10.1016/S1836-9553(10)70041-2

[17] Ramdharry, G.M., Day, B.L., Reilly, M.M. and Marsden, J.F. (2012) Foot Drop Splints Improve Proximal as Well as Distal Leg Control during Gait in Charcout-Marie-Tooth Disease. Muscle Nerve, 46, 512-519. https://doi.org/10.1002/mus.23348

[18] Kozhevnikov, O.V., Kosov, I.S., Ivanov, A.V., Gribova, I.V. and Bolotov, A.V. (2015) Comparative Evaluation of the Results of Complex and Conservative Treatment of Flat-Calf Deformities of the Feet in Children. Bulletin of Traumatology and Orthopedics, 2, 55-59.

[19] Mnatsakanian, A., Kissel, J.T., Terry, P. and King, W.M. (2017) One Clinic's Experience with Carbon Fiber Orthoses in Neuromuscular Disease. Muscle and Nerve, 2 , 202-205. https://doi.org/10.1002/mus.25233

[20] Uygur, F., Bek, N., Kuklu, B. and Yilmaz, O. (2001) Orthotic Management of the Lower Limb in Children with Hereditary Motor Sensory Neuropathy (NMSN). Prosthetics and Orthotics International, 25, 139-143. https://doi.org/10.1080/03093640108726587

[21] Anens, E., Emtner, M. and Hellstrom, K. (2015) Exploratory Study of Physical Activity in Persons with Charcot-Marie-Tooth Disease. Archives of Physical Medicine and Rehabilitation, 2, 260-268. https://doi.org/10.1016/j.apmr.2014.09.013

[22] Phillips, M.F., Robertson, Z., Killen, B. and White, B. (2012) A Pilot Study of a Ros- 
sover Trial with Randomized Use of Ankle-Foot Orthoses for People with Charcot-Marie-Tooth Disease. Clinical Rehabilitation, 9, 534-544. https://doi.org/10.1177/0269215511426802

[23] Rose, K.J., Hiller, C.E., Mandarakas, M., Raymond, J., Refshauge, K. and Burns, J. (2015) Correlates of Functional Ankle Instability in Children and Adolescents with Charcot-Marie-Tooth Disease. Journal of Foot and Ankle Research, 8, 61. https://doi.org/10.1186/s13047-015-0118-1

[24] Wegener, C., Wegener, K., Smith, R., Schott, K.H. and Burns, J. (2015) Biomechanical Effects of Sensorimotor Orthoses in Adults with Charcot-Marie-Tooth Disease. Journal of Foot and Ankle Research, 2, 39. https://doi.org/10.1186/1757-1146-8-S2-O39

[25] LoPiccolo, M., Chilvers, M., Graham, B. and Manoli, A. (2010) Effectiveness of the Cavus Foot Orthosis. Journal of Surgical Orthopaedic Advances, 3, 166-169.

[26] Ortlieb, A., Olivier, J., Bouri, M., Bleuler, H. and Kuntzer, T. (2015) From Gait Measurements to Design of Assistive Orthoses for People with Neuromuscular Diseases. Proceedings of the IEEE $\backslash$ RAS-EMBS International Conference on Rehabilitation Robotics, Singapore, 11-14 August 2015, 368-373. https://doi.org/10.1109/ICORR.2015.7281227

[27] Maynou, C., Szymanski, C. and Thiounn, A. (2017) The Adult Cavus Foot. EFORT Open Reviews, 2, 221-229. https://doi.org/10.1302/2058-5241.2.160077

[28] Georgiev, H. and Georgiev, G.P. (2014) Talectomy for Equinovarus Deformity in Family Members with Hereditary Motor and Sensory Neuropathy Type I. Case Reports in Orthopedics, 2014, Article ID: 643480. https://doi.org/10.1155/2014/643480

[29] Zhou, Y., Zhou, B., Liu, J., Tan, X., Tao, X., Chen, W. and Tang, K. (2014) A Prospective Study of Midfoot Osteotomy Combined with Adjacent Joint Sparing Internal Fixation in Treatment of Rigid Pes Cavus Deformity. Journal of Orthopaedic Surgery and Research, 9, 44. https://doi.org/10.1186/1749-799X-9-44

[30] Hoyle, J.C., Isfort, M.C., Roggenbuck, J. and Arnold, W.D. (2015) The Genetics of Charcot-Marie-Tooth Disease: Current Trend Sand Future Implications for Diagnosis and Management. The Application of Clinical Genetics, 8, 235-243.

[31] Chetlin, R.D., Gutmann, L. and Tarnopolsky, M. (2004) Resistance Training Effectiveness in Patients with Charcot-Marie-Tooth Disease: Recommendations for Exercise Prescription. Archives of Physical Medicine and Rehabilitation, 8, 1217-1223. https://doi.org/10.1016/j.apmr.2003.12.025

[32] Vinci, P. and Gargiulo, P. (2008) Poor Compliance with Ankle-Foot Orthoses in Charcot-Marie-Tooth Disease. European Journal of Physical and Rehabilitation Medicine, 1, 27-31.

[33] Shnayder, N.A., Goncharova, S.I., Duzackova, A.V., Popova, T.E., Dmitrenko, D.V., Kantimirova, E. and Shevchenko, S.A. (2015) Supposed Barriers and Motivators to Physical Activity in Patients with Charcot-Marie-Tooth Disease. Siberian Medical Review, 2, 15-25. https://doi.org/10.20333/25000136-2015-2-15-25

[34] Shnayder, N.A., Petryaeva, O.V., Artyukhov, I.P., Sapronova, M.R. and Loginova, I.O. (2017) A Rare Case of Charcot-Mari-Tooth Disease Type 2S in a 20-Year-Old Man. International Journal of Biomedicine, 4, 324-326.

https://doi.org/10.21103/Article7(4)_CR1

[35] Kalane, U.D., Datar, C. and Mahadevan, A. (2015) First Reported Case of Charcot Marie Tooth Disease Type 4C in a Child from India with SH3TC2 Mutation But Absent Spinal Deformities. Neurology India, 3, 395-398.

https://doi.org/10.4103/0028-3886.158222 
[36] The Social Network for Communicating People with CMT, Russia. https://vk.com/cmtarus

[37] A Patient Network and Real-Time Research Platform, USA. https://www.patientslikeme.com/patients

[38] CMT Support Group and Discussion Community, USA. https://www.inspire.com/groups/charcot-marie-tooth-cmt/

[39] CMT Support Group. https://www.dailystrength.org/group/charcot-marie-tooth-disease

[40] The Alliance of Rare Diseases, France. http://forums.maladiesraresinfo.org/maladie-charcot-marie-tooth-f200.html

[41] The Charcot-Marie-Tooth Association, USA. https://www.cmtausa.org/index.php

[42] Danilov, D.S. (2008) Individual Choice of Modern Pharmacotherapy of Schizophrenia (Basic Principles, Discussion of the Results of Clinical Studies and Some Practical Recommendations). Psychiatry and Pharmacotherapy, 10, 50-57.

[43] Gluschenko, E.V., Shnayder, N.A., Kantimirova, E., Kozulina, E.A., Voevoda, M.I., Maximov, V. and Allakhverdyan, A.A. (2012) Experience in Organizing of Diagnostic and Medical and Social Care to Patients with Hereditary Neuropathy Charcot-Marie-Tooth in the Krasnoyarsk Region. Neuromuscular Disease, 1, 41-53.

[44] Gluschenko, E.V. (2012) Case of Hereditary Motor-Sensory Neuropathy Charcot-MarieTooth of Type 1. Siberian Medical Review, 4, 84-87.

[45] Cramer, J.A., Scheyer, R.D. and Mattson, R.H. (1990) Compliance Declines between Clinic Visits. Archives of Internal Medicine, 7, 1509-1510. https://doi.org/10.1001/archinte.1990.00390190143023

[46] Naumova, E.A. and Schvartc, Yu.G. (2006) Performing by Patients the Medical Prescriptions: Whether Effective Are Interventions Aimed to Improve This Indicator. International Journal of Medical Practice, 1, 48-60.

[47] Schnayder, N.A., Gluschenko, E.V. and Kozulina, E.A. (2011) Assessment of the Life Quality in Patients with Hereditary Neuropathy Charcot Marie-Tooth in the Krasnoyarsk Region. Bulletin of Siberian Medicine, 2, 57-62.

[48] Vinci, P., Gargiulo, P., Panunzi, M. and Baldini, L. (2009) Psychological Distress in Patients with Charcot-Marie-Tooth Disease. European Journal of Physical and Rehabilitation Medicine, 3, 385-389. 